\title{
Does Chinese calligraphy therapy reduce neuropsychiatric symptoms: a systematic review and meta-analysis
}

\author{
Kuan-Yu Chu ${ }^{1,2,3^{*}}$, Chih-Yang Huang ${ }^{2}$ and Wen-Chen Ouyang ${ }^{4,5}$
}

\begin{abstract}
Background: There are currently no systematic reviews or meta-analyses of Chinese calligraphy therapy (CCT) to reduce neuropsychiatric symptoms. The aim of this systematic review and meta-analysis was to explore the efficacy of CCT for people with neuropsychiatric symptoms.

Methods: We searched Chinese and English databases, including the Cochrane Central Register of Controlled Trials and Wanfang Data for relevant articles published between the earliest year available and December 2016. The search was limited to randomized controlled trials and controlled clinical studies and the associated keywords were "handwriting," "Chinese calligraphy," "Chinese calligraphy therapy," "Calligraphy exercise," and "Calligraphy training." The 21 articles that met these criteria were used in the analysis. The Joanna Briggs Institute critical appraisal checklist was used to assess methodological quality.

Results: CCT significantly reduced psychosis (10 studies, 965 subjects, standardized mean difference $[S M D]=-0.17,95 \%$ confidence intervals $[C I][-0.30,-0.40], Z=2.60, p<0.01$ ), anxiety symptoms (9 studies, 579 subjects, $\mathrm{SMD}=-0.78,95 \% \mathrm{Cl}[-0.95,-0.61], Z=8.98, p<0.001$ ), and depressive symptoms (7 studies, 456 subjects, $S M D=-0.69,95 \% \mathrm{Cl}[-0.88,-0.50], Z=7.11, p<0.001)$. CCT also significantly improved cognitive function ( 2 studies, 55 subjects, $\mathrm{MD}=2.17,95 \% \mathrm{Cl}[-0.03,4.38], \mathrm{Z}=1.93, p=0.05$ ) and neurofeedback (3 studies, 148 subjects, SMD $=-1.09,95 \% \mathrm{Cl}[-1.44,-0.73], Z=6.01, p<0.001$ ). The therapy also significantly reduced the positive psychopathological expression of schizophrenia symptoms (4 studies, 287 subjects, $\mathrm{SMD}=-0.35,95 \% \mathrm{Cl}[-0.59,-0.12], \mathrm{Z}=2.96, p=0.003)$ and reduced the negative symptoms of schizophrenia (4 studies, 276 subjects, SMD $=-1.39,95 \% \mathrm{Cl}[-1.65,-1.12], Z=10.23, p<0.001$ ).
\end{abstract}

Conclusions: CCT exerts a curative effect on neuropsychiatric symptoms, but the evidence remains insufficient. A large number of RCTs are needed to facilitate additional systematic reviews of evidence for CCT.

Keywords: Chinese calligraphy, Art therapy, Psychiatric disorders

\section{Background}

The term psychosis refers to a serious psychological disorder characterized by obvious and long-lasting abnormalities in understanding, emotion, cognition, behavior, and other psychological activities. Psychoses such as schizophrenia often involve cognitive impairment and comorbid anxiety and depression [1]. Pharmacological therapy often has a limited effect or produces side

\footnotetext{
* Correspondence: kyc032@gmail.com

${ }^{1}$ Taoyuan General Hospital, Taoyuan City, Taiwan

${ }^{2}$ Department of Fine Art \& Culture Creative Design, Hua-Fan University, New

Taipei City, Taiwan

Full list of author information is available at the end of the article
}

effects [2, 3]. Non-pharmacological therapies (such as psychotherapy, occupational therapy, and art therapy) for patients with psychiatric disorders are useful, adaptable, and potentially cost-effective approaches to improve outcomes and quality of life [4].

Chinese calligraphy therapy (CCT) is a branch of art therapy that involves visual-spatial patterning of characters. This type of calligraphy is more than an art therapy; in essence, it involves culture, health, behaviour treatment and rehabilitation. The art nature is only one of its varied roles and functions. It necessitates exercising motor control of the brush to follow 
specific character configurations based on a projection of the cognitive images of the characters [5]. CCT requires the use of a soft-tipped brush to reproduce Chinese glyphs. It combines physical, mental, and personal processes and integrates visual performance, spatial abilities, and cognitive planning [6].

Recent empirical studies have shown that the practice of calligraphy may improve behavioral and psychosomatic disorders and may have a therapeutic effect on attention and emotional stability [7]. CCT has been scientifically investigated within the contexts of psychology, cognitive science, and cognitive neuroscience, and the findings suggest that it can reduce neuropsychiatric symptoms [8].

There have been many systematic reviews of other art therapies, such as painting therapy, music therapy, and gardening therapy. However, there are no systematic reviews or meta-analyses of CCT's effect in reducing neuropsychiatric symptoms. Thus, the aim of this systematic review and meta-analysis was to explore the efficacy of CCT for people with neuropsychiatric symptoms.

\section{Methods}

\section{Search strategy}

A systematic search for articles was made in December 2016 using the databases MEDLINE, EMBASE, PsycINFO, Cochrane Central Register of Controlled Trials, and Wanfang Data. We searched for keywords and/or controlled vocabulary, such as medical subject headings and Emtree terms. Keywords were "handwriting," "Chinese calligraphy," "Chinese calligraphy therapy," "Calligraphy exercise," and "Calligraphy training."

\section{Study selection}

We included in the analysis studies that met the following criteria: (1) randomized controlled trial (RCT), cohort study, or case-control study, (2) published in Chinese or English, (3) subjects were either healthy or psychiatric patients, (4) experimental group intervention measures for Chinese calligraphy; the control group intervention could be general care, health education, or no care, and (5) measurement of physiological or mental indicators. The exclusion criteria were as follows: (1) not relevant to Chinese calligraphy/calligraphy therapy, (2) commentary, case report, or a review article, (3) experimental interventions other than calligraphy treatment, (4) and lack of a control group and/or overlapping populations.

\section{Quality assessment}

We used the guideline suggested by Queen's Joanna Briggs Collaboration critical appraisal checklist, Version 4.0 [9]. This scale contains 10 appraisal criteria that assess whether the assignment to treatment groups was truly random, participants were blinded to treatment allocation, allocation to treatment groups was concealed from the allocator, the outcomes of people who withdrew were described and included in the analysis, those assessing the outcomes were blind to the treatment allocation, the control and treatment groups were comparable at entry, the groups were treated identically other than for the named interventions, the outcomes were

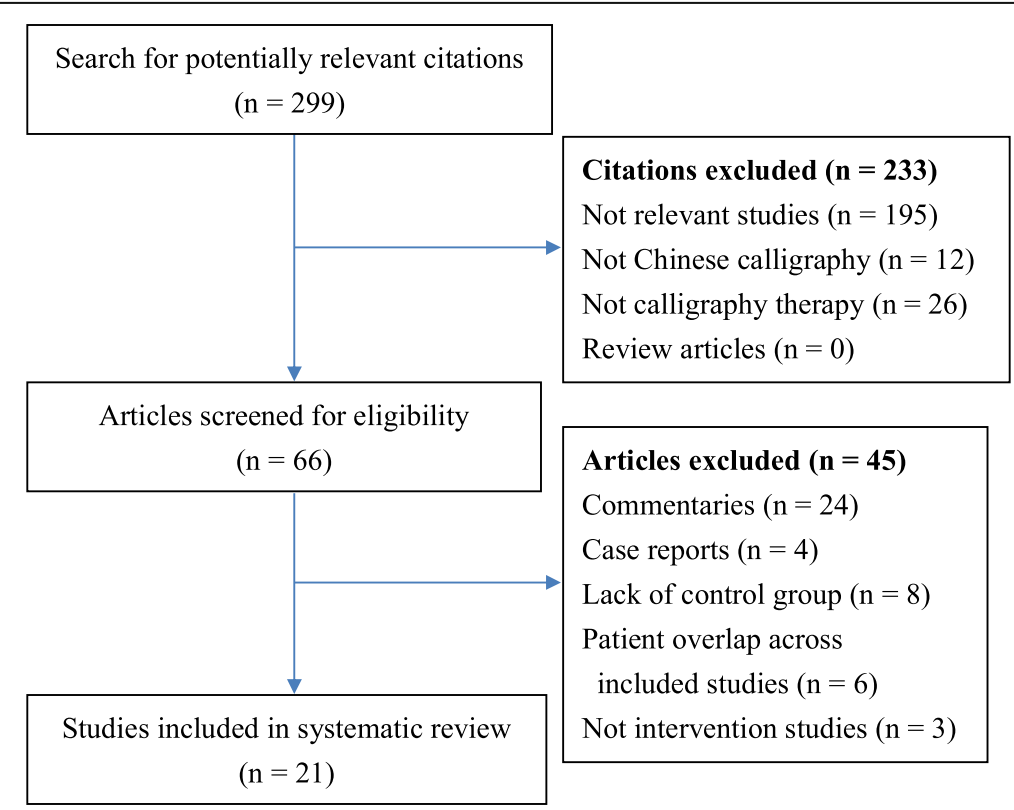

Fig. 1 Flow diagram of study selection 


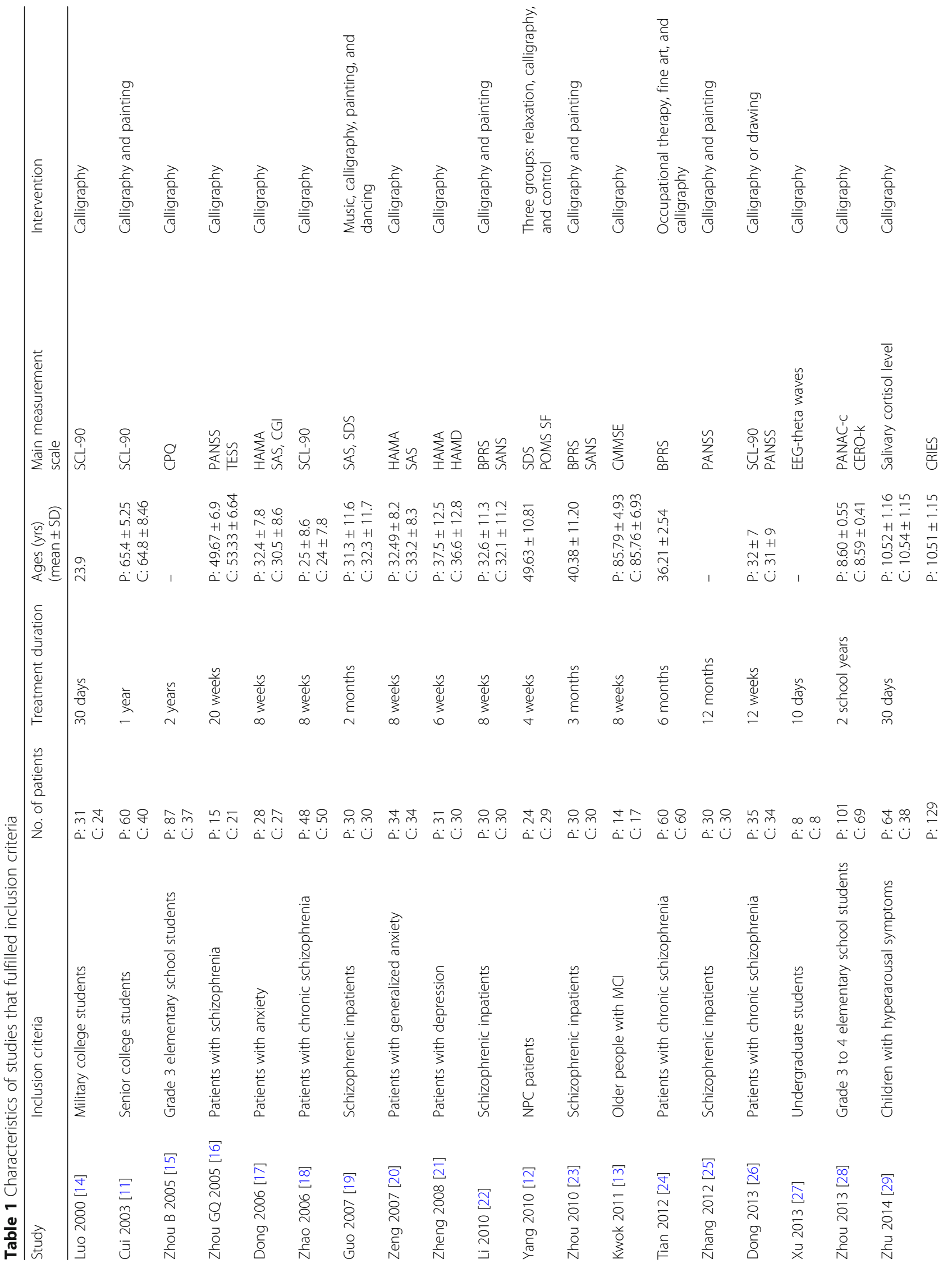




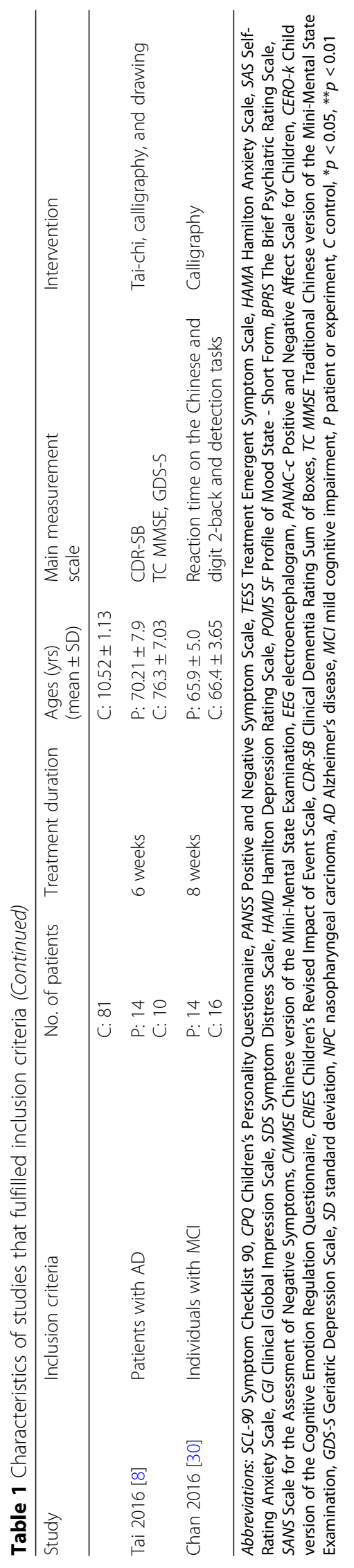




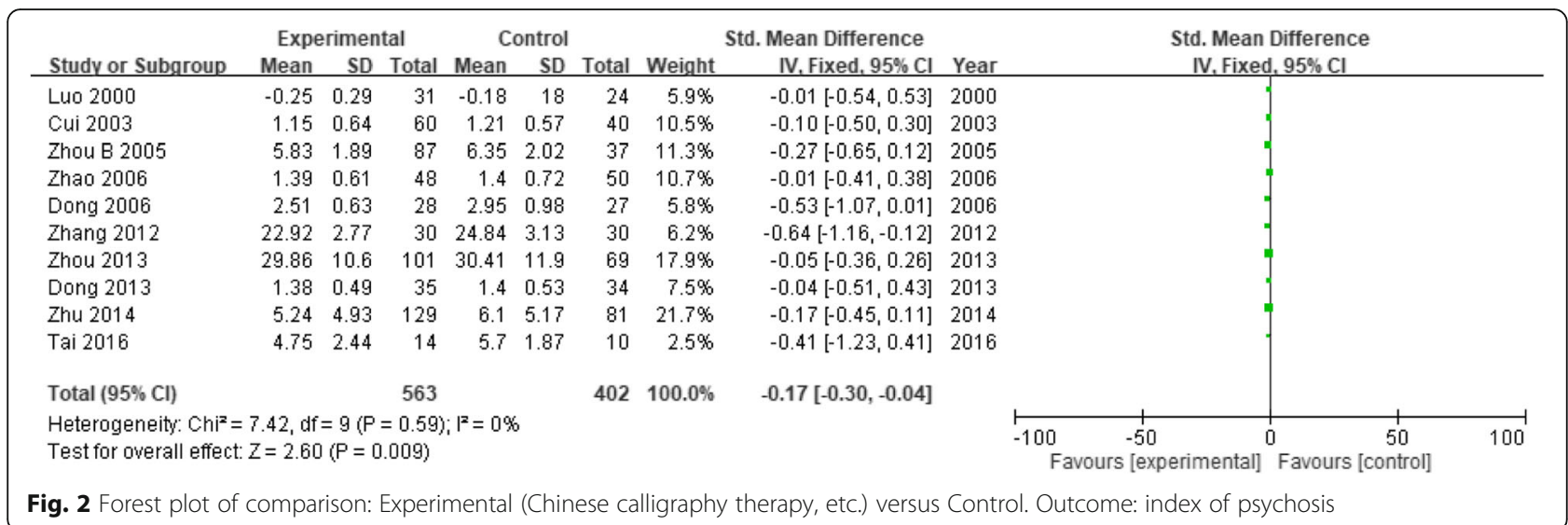

measured in the same way for all groups, the outcomes were measured in a reliable way, and the statistical analysis was appropriate. Two researchers extracted information and screened the quality of the articles independently. A third researcher was used to determine the quality of the studies in cases where it was difficult to reach a consensus.

\section{Data analysis}

Meta-analysis was performed using RevMan 5.3 software (Review Manager (RevMan) [Computer program]. Version 5.3. Copenhagen: The Nordic Cochrane Centre, The Cochrane Collaboration, 2014.). The mean and standard deviation of each group was calculated to determine the overall effect of the intervention. Chi-square was used to determine heterogeneity. In addition, I2 values were calculated to test the heterogeneity among the studies. When I2 was $<50 \%$, a fixed-effects model was used to determine the homogeneity among studies [10]. However, if there were differences between the studies (such as study location, population, and intervention program), a random-effects model analysis was used to avoid underestimation of treatment variability. Odds ratios and mean differences were used to compare different measurement indexes, to obtain standardized mean differences (SMD), and to estimate the combined effect level. Based on the different psychiatric symptoms and diseases included in the studies, we divided the metaanalysis into six groups: group 1: psychosis, group 2: anxiety, group 3: depression, group 4: cognition, group 5: neurofeedback, and group 6: schizophrenia.

\section{Results}

\section{Characteristics of the studies}

Figure 1 shows the process of study selection. Our initial search strategy yielded 299 citations, 233 of which were ineligible based on our screening of titles and abstracts. Thus, we retrieved the full text of 66 studies. Of these, 24 were excluded because they were commentaries; 4 were case reports; 8 lacked a control group; 6 had study populations that overlapped with other included studies; and 3 did not meet the eligibility criteria because they were not intervention studies. Consequently, 21 eligible studies were analyzed.

Table 1 shows study characteristics and patient demographic data from each of the 21 studies included in the review. These studies were published between 2000 and 2016, and had sample sizes ranging from 16 to 224. Ten studies measured psychosis or general psychosis (the meta-analysis results and forest

\begin{tabular}{|c|c|c|c|c|c|c|c|c|c|c|c|}
\hline \multirow[b]{2}{*}{ Studv or Subgroup } & \multicolumn{3}{|c|}{ Experimental } & \multicolumn{3}{|c|}{ Control } & \multicolumn{3}{|c|}{ Std. Mean Difference } & \multirow{2}{*}{\multicolumn{2}{|c|}{$\begin{array}{l}\text { Std. Mean Difference } \\
\text { IV, Fixed, } 95 \% \mathrm{Cl}\end{array}$}} \\
\hline & Mean & SD & Total & Mean & SD & Total & Weight & IV, Fixed, 95\% Cl & Year & & \\
\hline Luo 2000 & -0.12 & 0.41 & 31 & 0.1 & 0.32 & 24 & $9.8 \%$ & $-0.58[-1.13,-0.04]$ & 2000 & & \\
\hline Zhao 2006 & 1.23 & 0.66 & 48 & 1.82 & 0.56 & 50 & $16.5 \%$ & $-0.96[-1.38,-0.54]$ & 2006 & & \\
\hline Dong 2006 & 10.86 & 6.98 & 28 & 14.58 & 8.65 & 27 & $10.1 \%$ & $-0.47[-1.00,0.07]$ & 2006 & & \\
\hline Guo 2007 & 41.61 & 9.12 & 30 & 49.31 & 9.28 & 30 & $10.4 \%$ & $-0.83[-1.35,-0.30]$ & 2007 & & \\
\hline Zeng $200 ?$ & 8.46 & 5.93 & 34 & 13.62 & 7.48 & 34 & $11.9 \%$ & $-0.76[-1.25,-0.26]$ & 2007 & & \\
\hline Zheng 2008 & 8.45 & 2.81 & 31 & 12.45 & 3.92 & 30 & $9.7 \%$ & $-1.16[-1.71,-0.62]$ & 2008 & & \\
\hline Yang 2009 & 3.5 & 1.69 & 24 & 4.04 & 1.54 & 29 & $9.8 \%$ & $-0.33[-0.88,0.21]$ & 2009 & & \\
\hline Li 2010 & 5.61 & 1.23 & 30 & 6.72 & 1.35 & 30 & $10.3 \%$ & $-0.85[-1.38,-0.32]$ & 2010 & & \\
\hline Dong 2013 & 1.24 & 0.65 & 35 & 1.83 & 0.58 & 34 & $11.6 \%$ & $-0.95[-1.45,-0.45]$ & 2013 & & \\
\hline Total $(95 \% \mathrm{Cl})$ & & & 291 & & & 288 & $100.0 \%$ & $-0.78[-0.95,-0.61]$ & & & \\
\hline $\begin{array}{l}\text { Heterogeneity: } \mathrm{Chi}^{2}= \\
\text { Test for overall effect }\end{array}$ & $\begin{array}{l}7.53, \mathrm{df} \\
Z=8.98\end{array}$ & $\begin{array}{l}=8 \stackrel{P}{ } \\
(P \leq 0\end{array}$ & $=0.48)$ & $i^{2}=0 \%$ & & & & & & $\begin{array}{cccc}\vdash & 1 & 1 & 1 \\
-100 & -50 & 0 & 50 \\
& \text { Favours [experimental] } & \text { Favours [control] }\end{array}$ & 100 \\
\hline
\end{tabular}




\begin{tabular}{|c|c|c|c|c|c|c|c|c|c|c|c|c|}
\hline \multirow[b]{2}{*}{ Studv or Subgroup } & \multicolumn{3}{|c|}{ Experimental } & \multicolumn{3}{|c|}{ Control } & \multicolumn{3}{|c|}{ Std. Mean Difference } & \multirow{2}{*}{\multicolumn{3}{|c|}{$\begin{array}{l}\text { Std. Mean Difference } \\
\text { IV, Fixed, } 95 \% \mathrm{Cl}\end{array}$}} \\
\hline & Mean & SD & Total & Mean & SD & Total & Weight & IV, Fixed, 95\% Cl & Year & & & \\
\hline Luo 2000 & -0.31 & 0.39 & 31 & -0.09 & 0.26 & 24 & $12.1 \%$ & $-0.64[-1.19,-0.09]$ & 2000 & & & \\
\hline Zhao 2006 & 1.49 & 0.71 & 48 & 1.83 & 0.65 & 50 & $22.3 \%$ & $-0.50[-0.90,-0.09]$ & 2006 & & & \\
\hline Guo 2007 & 42.32 & 9.21 & 30 & 50.58 & 8.84 & 30 & $12.7 \%$ & $-0.90[-1.44,-0.37]$ & 2007 & & & \\
\hline Zheng 2008 & 7.2 & 4.32 & 31 & 11.4 & 5.42 & 30 & $13.1 \%$ & $-0.85[-1.37,-0.32]$ & 2008 & & & \\
\hline Yang 2009 & 3.79 & 1.91 & 24 & 5.76 & 2.78 & 29 & $11.4 \%$ & $-0.80[-1.36,-0.24]$ & 2009 & & & \\
\hline Li 2010 & 5.61 & 1.23 & 30 & 6.72 & 1.35 & 30 & $12.8 \%$ & $-0.85[-1.38,-0.32]$ & 2010 & & & \\
\hline Dong 2013 & 1.5 & 0.72 & 35 & 1.84 & 0.67 & 34 & $15.7 \%$ & $-0.48[-0.96,-0.00]$ & 2013 & & & \\
\hline Total $(95 \% \mathrm{Cl})$ & & & 229 & & & 227 & $100.0 \%$ & $-0.69[-0.88,-0.50]$ & & & & \\
\hline $\begin{array}{l}\text { Heterogeneity: } \mathrm{Chi}^{2}= \\
\text { Test for overall effect: }\end{array}$ & $\begin{array}{l}3.09, \mathrm{df}= \\
Z=7.11\end{array}$ & $\begin{array}{l}=6(P= \\
(P \subset 0\end{array}$ & $\begin{array}{l}=0.80) \\
.00001\end{array}$ & $i^{2}=0 \%$ & & & & & & $\begin{array}{ccc}\vdash & 1 \\
-100 & -50 & 1 \\
& \text { Favours [experimental] }\end{array}$ & $\begin{array}{c}50 \\
\text { Favours [control] }\end{array}$ & 100 \\
\hline
\end{tabular}

map are shown in Fig. 2). Nine studies on anxiety were analyzed; one study was removed [11] because the sensitivity analysis indicated large heterogeneity (Fig. 3). Seven studies on depression were analyzed (Fig. 4). Two studies on cognitive impairment were analyzed (Fig. 5) and three studies on neurofeedback were analyzed (Fig. 6).

Eight studies evaluated patients with schizophrenia, of which four measured positive psychopathology of schizophrenia and four measured negative syndromes of schizophrenia (see Figs. 7 and 8 ).

Table 2 shows the methodological quality of the 21 included studies. Two studies clearly documented the use of random allocation [12, 13]. The remaining quality criteria were scored based on the narrative of the studies. The scores for risk of bias ranged from 6 to 9 points. Agreement between the two reviewers was assessed using Cohen's kappa coefficient and was $0.891(p<0.001)$, indicating a high degree of consistency.

Figure 2 shows the meta-analysis results for group 1. Using a fixed-effects model, the heterogeneity test (I2) result for the seven studies was $0 \%$, indicating homogeneity among the studies. The SMD was used to estimate the combined effect of measurements using different scales. The results showed that CCT can significantly reduce psychosis (10 studies, 965 subjects, SMD $=-0.17,95 \%$ CI $[-0.30,-0.04], \mathrm{Z}=$ 2.60, $p=0.009$ ).

Figure 3 shows the meta-analysis results for group 2 . Using a fixed-effects model, the heterogeneity test (I2) was $0 \%$, indicating that the nine studies were homogeneous. The SMD indicated that calligraphy treatment significantly reduced anxiety symptoms (9 studies, 579 subjects, SMD $=-0.78,95 \% \mathrm{CI}[-0.95,-0.61], \mathrm{Z}=8.98$, $p<0.001)$.

Figure 4 shows the meta-analysis results for group 3. Using a fixed-effects model, the heterogeneity test (I2) was $0 \%$, indicating that the seven studies were homogeneous. The SMD indicated that calligraphy treatment significantly reduced depressive symptoms (7 studies, 456 subjects, SMD $=-0.69,95 \% \mathrm{CI}[-0.88,-0.50], \mathrm{Z}=7.11, p<0.001)$. Figure 5 shows the meta-analysis results for group 4 . Using a fixedeffects model, the heterogeneity test (I2) was $0 \%$, indicating that the two studies were homogeneous. The estimated combined effect showed that CCT significantly improved cognitive function (2 studies, 55 subjects, $\mathrm{MD}=2.17,95 \% \mathrm{CI}$ $[-0.03,4.38], \mathrm{Z}=1.93, p=0.05)$. Figure 6 shows the metaanalysis results for group 5 . Using a fixed-effects model, the heterogeneity test (I2) was $0 \%$, indicating that the three studies were homogeneous. The estimated combined effect showed that CCT significantly improved neurofeedback (3 studies, 148 subjects, SMD $=-1.09,95 \%$ CI $[-1.44,-0.73$, $\mathrm{Z}=6.01, p<0.001)$. Figure 7 shows the meta-analysis results for group 6-1 (schizophrenia-psychopathy). Using a fixedeffects model, the heterogeneity test (I2) was $0 \%$, indicating that the four studies were homogeneous. The estimated combined effect showed that CCT significantly reduced positive psychopathological expression of schizophrenia symptoms (4 studies, 287 subjects, SMD $=-0.35,95 \%$ CI $[-0.59$, -0.12 ], $Z=2.96, p=0.003)$. Figure 8 shows the metaanalysis results for group 6-2 (schizophrenia-negative

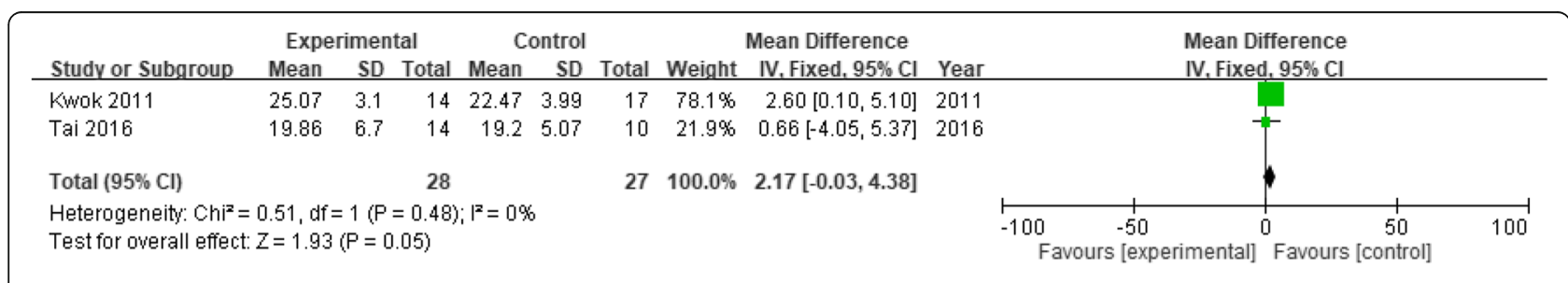

Fig. 5 Forest plot of comparison: Experimental (Chinese calligraphy therapy, etc.) versus Control. Outcome: index of cognitive function 


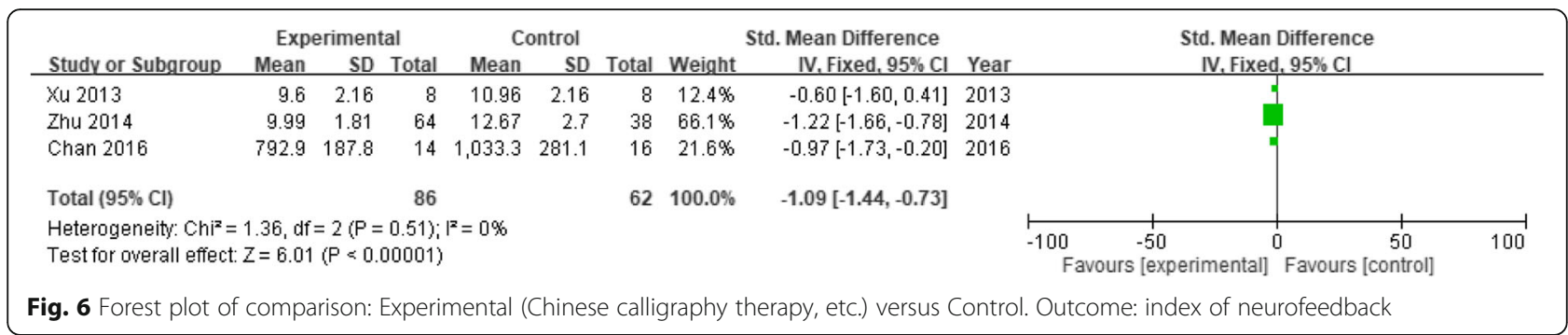

syndrome). Using a fixed-effects model, the heterogeneity test (I2) was 15\%, indicating that the four studies were homogeneous. The estimated combined effect showed that CCT significantly reduced negative symptoms of schizophrenia (4 studies, 276 subjects, $\mathrm{SMD}=-1.39,95 \% \mathrm{CI}[-1.65$, 1.12], $\mathrm{Z}=10.23, p<0.001)$.

\section{Discussion}

The purpose of this study was to explore the effectiveness of calligraphy therapy in improving symptoms of psychiatric disorders by reviewing and analyzing relevant literature. Twenty-one studies met the inclusion criteria and were reviewed. Most of the evidence suggests that CCT can change targeted behaviors in individuals with neuropsychiatric symptoms and that CCT is associated with improvements in objective measurements of psychiatric performance.

Evidence from these kinds of studies is needed before CCT can be considered effective for neuropsychiatric symptoms. The study findings reviewed here suggest that, used as psychiatric therapy, CCT can significantly improve selected neuropsychiatric symptoms. The use of CCT in compensation-focused interventions and selected psychotherapeutic interventions may lead to neuropsychiatric changes and thus improve daily life.

RCTs provide the best evidence of the efficacy of CCT. In RCTs with large samples, there is more balance between the characteristics of participants in the treatment and control groups. In small-sample RCTs, some characteristics may not be balanced between groups. Table 1 shows that most of the sample sizes in each group were between 15 and 30. Only about half of the included studies had quality scores greater than 8 (Table 2). Therefore, more high-quality RCTs are needed to strengthen the evidence for CCT's effect in reducing neuropsychiatric symptoms. If more systematic reviews are produced to establish clinical guidelines, this could increase the clinical use of CCT.

A funnel plot of the CCT literature was symmetrical (i.e., showed no positive or negative relations between effect size and standard error). This indicates that there was no publication error. The best way to avoid publication bias is to begin with a rigorous examination of the literature.

As this is the first review of the efficacy of CCT for neuropsychiatric symptoms, we are unable to compare the findings with other similar studies. Compared with other non-pharmacological therapies like music therapy, painting therapy, or gardening therapy, CCT is perhaps a more culture-specific therapy, as it requires participants to learn Chinese writing and use a special soft calligraphy brush.

Our findings show that calligraphy therapy can significantly enhance cognitive function and relieve neuropsychiatric and depressive symptoms. However, there were some study limitations. First, studies varied markedly in their intervention approaches and selected outcome measures, and were frequently hampered by design limitations. Second, the pattern of effects on specific neuropsychiatric domains was inconsistent across studies. Additionally, some important outcomes, such as daily functioning, quality of life, and neuropsychiatric symptom severity, were assessed infrequently in the reviewed studies. Moreover, handwritten communication has largely been replaced by typed communications

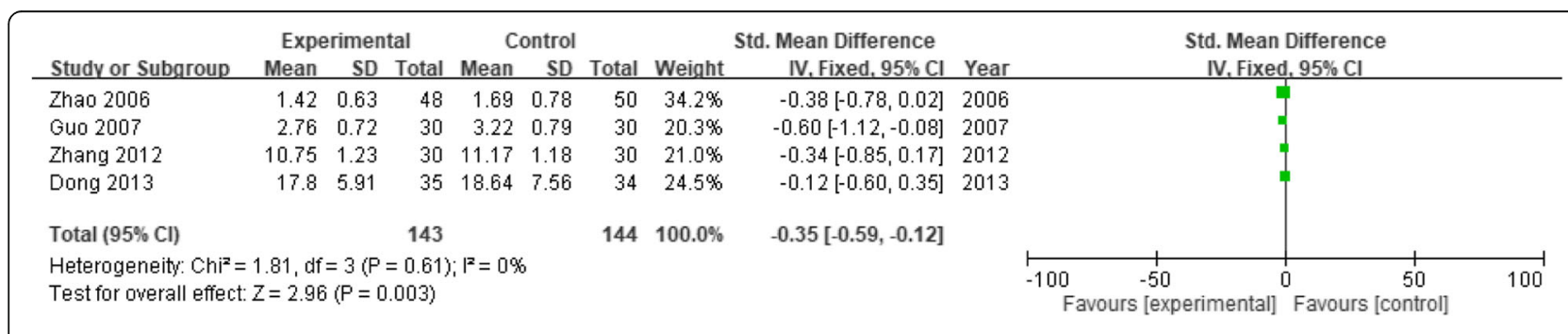

Fig. 7 Forest plot of comparison: Experimental (Chinese calligraphy therapy, etc.) versus Control. Outcome: index of schizophrenia-psychopathy 


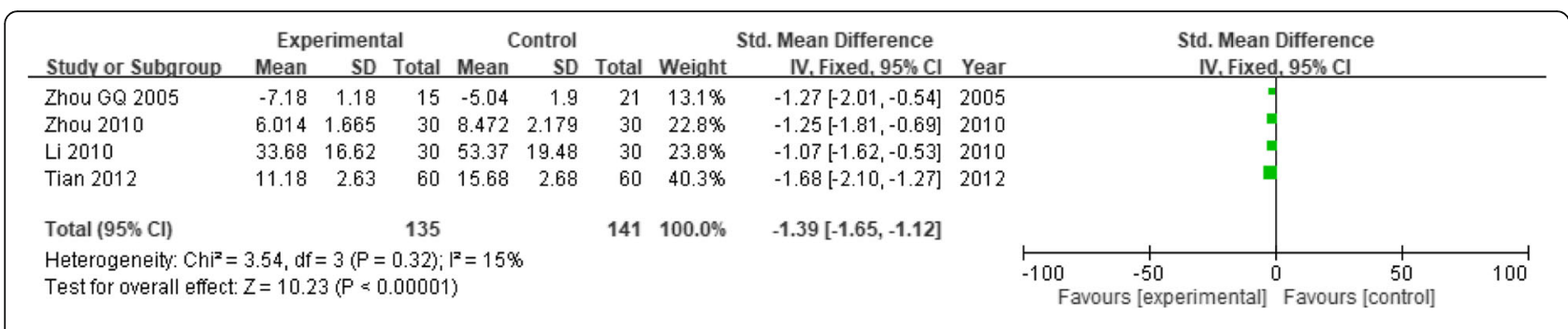

Fig. 8 Forest plot of comparison: Experimental (Chinese calligraphy therapy, etc.) versus Control. Outcome: index of schizophrenia-negative syndrome

(and, in more recent years, by mobile phone calls and texts). This may have affected the results.

Overall, the results from trials are promising but inconclusive. Additional well-designed and adequately powered trials are warranted. However, this evidence

Table 2 Methodological quality assessment of the included studies (JBI)

\begin{tabular}{|c|c|c|c|c|c|c|c|c|c|c|c|c|}
\hline \multirow[t]{2}{*}{ Study } & \multirow{2}{*}{$\begin{array}{l}\text { Study } \\
\text { design }\end{array}$} & \multicolumn{10}{|c|}{ Score criteria } & \multirow[b]{2}{*}{ Total } \\
\hline & & 1 & 2 & 3 & 4 & 5 & 6 & 7 & 8 & 9 & 10 & \\
\hline Luo 2000 & CCS & 0 & 0 & 0 & 1 & 1 & 1 & 1 & 1 & 1 & 1 & 7 \\
\hline Cui 2003 & CCS & 0 & 0 & 0 & 1 & 1 & 1 & 1 & 1 & 1 & 1 & 7 \\
\hline Zhou B 2005 & $\mathrm{CS}$ & 0 & 0 & 0 & 1 & 0 & 1 & 1 & 1 & 1 & 1 & 6 \\
\hline Zhou GQ 2005 & CCS & 0 & 0 & 0 & 1 & 1 & 1 & 1 & 1 & 1 & 1 & 7 \\
\hline Dong 2006 & $\mathrm{RCT}$ & 1 & 1 & 1 & 0 & 1 & 1 & 1 & 1 & 1 & 1 & 9 \\
\hline Zhao 2006 & $\mathrm{RCT}$ & 1 & 1 & 0 & 1 & 1 & 1 & 1 & 1 & 1 & 1 & 9 \\
\hline Guo 2007 & $\mathrm{RCT}$ & 1 & 1 & 0 & 0 & 1 & 1 & 1 & 1 & 1 & 1 & 8 \\
\hline Zeng 2007 & $\mathrm{RCT}$ & 1 & 1 & 0 & 0 & 1 & 1 & 1 & 1 & 1 & 1 & 8 \\
\hline Zheng 2008 & $\mathrm{RCT}$ & 1 & 1 & 0 & 0 & 1 & 1 & 1 & 1 & 1 & 1 & 8 \\
\hline Li 2010 & $\mathrm{RCT}$ & 1 & 1 & 0 & 0 & 1 & 1 & 1 & 1 & 1 & 1 & 8 \\
\hline Yang 2010 & $\mathrm{RCT}$ & 1 & 1 & 0 & 1 & 1 & 1 & 1 & 1 & 1 & 1 & 9 \\
\hline Zhou 2010 & $\mathrm{RCT}$ & 1 & 1 & 0 & 0 & 1 & 1 & 1 & 1 & 1 & 1 & 8 \\
\hline Kwok 2011 & $\mathrm{RCT}$ & 1 & 0 & 0 & 0 & 1 & 1 & 1 & 1 & 1 & 1 & 6 \\
\hline Tian 2012 & $\mathrm{RCT}$ & 1 & 1 & 0 & 1 & 1 & 1 & 1 & 1 & 1 & 1 & 9 \\
\hline Zhang 2012 & CCS & 1 & 0 & 0 & 0 & 1 & 1 & 1 & 1 & 1 & 1 & 7 \\
\hline Dong 2013 & $\mathrm{RCT}$ & 1 & 1 & 1 & 0 & 1 & 1 & 1 & 1 & 1 & 1 & 9 \\
\hline Xu 2013 & CCS & 0 & 0 & 0 & 0 & 1 & 1 & 1 & 1 & 1 & 1 & 6 \\
\hline Zhou 2013 & CS & 0 & 0 & 0 & 1 & 0 & 1 & 1 & 1 & 1 & 1 & 6 \\
\hline Zhu 2014 & $\mathrm{RCT}$ & 1 & 1 & 0 & 0 & 1 & 1 & 1 & 1 & 1 & 1 & 8 \\
\hline Tai 2016 & CCS & 0 & 0 & 0 & 0 & 1 & 1 & 1 & 1 & 1 & 1 & 6 \\
\hline Chan 2016 & $\mathrm{RCT}$ & 1 & 1 & 0 & 0 & 1 & 1 & 1 & 1 & 1 & 1 & 8 \\
\hline
\end{tabular}

Abbreviations: JBI Joanna Briggs Institute, $R C T$ randomized controlled trial, CCS controlled clinical study, CS cohort study

Score criteria: 1 . Was the assignment to treatment groups truly random? 2. Were participants blinded to treatment allocation? 3 . Was allocation to treatment groups concealed from the allocator? 4 . Were the outcomes of people who withdrew described and included in the analysis? 5 . Were those assessing the outcomes blind to the treatment allocation? 6. Were control and treatment groups comparable at entry? 7. Were groups treated identically other than for the named interventions? 8 . Were outcomes measured in the same way for all groups? 9. Were outcomes measured in a reliable way? 10. Was appropriate statistical analysis used?

Score descriptions: yes $=1, n o=0$, unclear $=0$, not applicable $=0$ must be treated with caution because of methodological limitations. To better assess the value of nonpharmacological interventions for this population, we recommend the following: (1) RCTs should have a large size of over 30 subjects; (2) standards for cognitive/ neuropsychiatric rehabilitation must be established for treatment of diseases, such as stroke, Alzheimer's disease and MCI; (3) general character templates for adaptation and modification in diverse fields of clinical trials must be standardized; (4) character form design must be set; (5) authority for standards of template designs, protocols for CCT and a large database for cases and trial follow ups must be established.

\section{Conclusion}

This study shows that CCT exerts a curative effect on neuropsychiatric symptoms, but the evidence remains insufficient. A large number of RCTs are needed to facilitate additional systematic reviews of evidence for CCT. In general, we hope that this paper offers a method for a systematic CCT review and meta-analysis, which may provide a basis for establishing standards for $\mathrm{CCT}$ in clinical trials and applications in the future.

\section{Additional files}

Additional file 1: AF1: Search terms (DOC $30 \mathrm{~kb}$ )

Additional file 2: List of studies excluded from the review by exclusion category (DOC $67 \mathrm{~kb}$ )

Acknowledgments

We thank Diane Williams, PhD, from Edanz Group (www.edanzediting.com) for editing a draft of this manuscript.

\section{Funding}

This project received funding from Taoyuan General Hospital under agreement no. PTH10534. The corresponding author had full access to all data in the study and had final responsibility for the decision to submit for publication. The sponsor of the study had no role in the study design, data collection, analysis, interpretation, or writing of the paper.

\section{Availability of data and materials}

All data generated or analyzed during this study are included in this published article and its Additional file 1 and Additional file 2. 


\section{Authors' contributions}

KYC and CYH designed the study and contributed substantially to the design of the search strategy. KYC and CYH searched the literature and extracted data. WCO performed the analysis and interpreted the data. KYC wrote the first draft of the manuscript and $\mathrm{CYH}$ and WCO critically reviewed the manuscript. All authors read and approved the final manuscript.

\section{Ethics approval and consent to participate}

Ethical approval was not required because of the nature of the study.

\section{Consent for publication}

Not applicable.

\section{Competing interests}

The authors declare that they have no competing interests.

\section{Publisher's Note}

Springer Nature remains neutral with regard to jurisdictional claims in published maps and institutional affiliations.

\section{Author details}

${ }^{1}$ Taoyuan General Hospital, Taoyuan City, Taiwan. ${ }^{2}$ Department of Fine Art \& Culture Creative Design, Hua-Fan University, New Taipei City, Taiwan. ${ }^{3}$ College of General studies, Yuan-Ze University, Taoyuan City, Taiwan. ${ }^{4}$ Jianan Psychiatric Center, Tainan City, Taiwan. ${ }^{5}$ Department of Psychiatry, College of Medicine, Kaohsiung Medical University, Kaohsiung, Taiwan.

Received: 3 July 2017 Accepted: 17 January 2018

Published online: 07 March 2018

\section{References}

1. American Psychiatric Association. Diagnostic and statistical manual of mental disorders. 5th (DSM-5 $\left.{ }^{\oplus}\right)$. Arlington, VA: American Psychiatric Association; 2013.

2. Bet PM, Hugtenburg JG, Penninx BW, Hoogendijk WJ. Side effects of antidepressants during long-term use in a naturalistic setting. Eur Neuropsychopharmacol. 2013;23:1443-51.

3. Samaras K, Correll CU, Mitchell AJ, De Hert M. Diabetes risk potentially underestimated in youth and children receiving antipsychotics. JAMA Psychiat. 2014;71:209-10.

4. Rodakowski J, Saghafi E, Butters MA, Skidmore ER. Non-pharmacological interventions for adults with mild cognitive impairment and early stage dementia: an updated scoping review. Mol Asp Med. 2015;43-44:38-53.

5. HSR K. Shufa: Chinese calligraphic handwriting $(\mathrm{CCH})$ for health and behavioural therapy. Int J Psychol. 2006;41:282-6.

6. HSR K, Zhu L, Chao AA, Chen HY, ICY L, Zhang M. Calligraphy and meditation for stress reduction: an experimental comparison. Psychol Res Behav Manag. 2014;7:47-52.

7. Zhou B. An experimental study on the influence of calligraphy exercises on children's behavior habits. Chinese calligraphy. Studies. 2007:4:72-80.

8. Tai SY, Hsu CL, Huang SW, Ma TC, Hsieh WC, Yang YH. Effects of multiple training modalities in patients with Alzheimer's disease: a pilot study. Neuropsychiatr Dis Treat. 2016;12:2843-9.

9. Aromataris, E., Fernandez, R. S., Godfrey, C., Holly, C., Khalil, H., \& Tungpunkom, P. Methodology for JBI umbrella reviews. 2014.

10. Higgins J, Thompson SG, Deeks JJ, Altman DG. Measuring inconsistency in meta-analyses. Br Med J. 2003:327:557-60.

11. Cui M, Ao X. The effect of calligraphy and painting exercise on mental health of senile college students. Sichuan. Ment Health. 2003;16:85-6.

12. Yang $\mathrm{XL}$, Li HH, Hong MH, HSR K. The effects of Chinese calligraphy handwriting and relaxation training in Chinese nasopharyngeal carcinoma patients: a randomized controlled trial. Int J Nurs Stud. 2010;47:550-9.

13. TCY K, Bai X, HSR K, JCY L, FKY H. Cognitive effects of calligraphy therapy for older people: a randomized controlled trial in Hong Kong. Clin Interv Aging. 2011;6:269-73

14. Luo Z, Miao DM, Gao D. The emotion regulation of calligraphy training to military college students. Psychol Sci. 2000;23:564-7.

15. Zhou B, Liu J, Sang B. The effect of calligraphy on the development of children's personality. Psychol Sci. 2005;28:1266-8.
16. Zhou GQ, Wang J, Zheng $H$. Effect of painting and drawing therapy in the mental functional rehabilitation of patients with schizophrenia. Chin J Clin Rehab. 2005;9:50-1.

17. Dong XP, Jia JM, Wang J, Zhang R. A control study of calligraphy training plus venlafaxine in the treatment of anxiety disorder. Chin J Behav Med Sci. 2006;5:027.

18. Zhao J, Deng W, Wen J. Intervention of calligraphy therapy on rehabilitation of chronic schizophrenia. Modern. Nursing. 2006;12:357-8.

19. Guo QF, Tian D, Zhao F. Effect of psychological rehabilitation on comprehensive art intervention for patient with schizophrenia. J Nurs Sci. 2007;22:13-5.

20. Zeng Z. The effect of calligraphy training in the treatment of patient with generalized anxiety disorder. Chin J Health Psychol. 2007;15:483-4.

21. Zheng G, Wang P, Liu XB. The effects of handwriting artist therapy to depression. Med J Chin People's Health. 2008;5:061.

22. Li YM, Li ZQ, Zhang Y. The effect of painting and calligraphy behavior therapy among the clinical rehabilitation of schizophrenia inpatients. Int J Nurs. 2010;29:189-92.

23. Zhou Q. The application of chess painting and calligraphy in the treatment of inpatients with schizophrenia. Straint. Pharm J. 2010;22:173-5.

24. Tian JH. Chronic schizophrenia individualized therapy clinical research. Psychiatrist. 2012;105:13-4.

25. Zhang Z, Bei CL, Wang YL. Effectiveness of handwriting and drawing practice for chronic phase schizophrenia. Med J Chin People's Health. 2012;24:2115-7.

26. Dong ZQ, Shi LY. Art behaviorial therapy for chronic schizophrenia intervention study. Gansu medical. Journal. 2013;32:38-40.

27. Xu M, Kao HSR, Zhang M, Lam SPW, Wang W. Cognitive-neural effects of brush writing of Chinese characters: cortical excitation of theta rhythm. Evid Based Complement Alternat Med. 2013;2013::11. https://doi.org/10.1155/ 2013/975190.

28. Zhou B, Tang J, Li C, Tang H, Li J. Positive effect of practicing Chinese calligraphic handwriting on emotional regulation strategies in children. J Psychol Sci. 2013;36:98-102.

29. Zhu Z, Wang R, HSR K, Zong Y, Liu Z, Tang S, et al. Effect of calligraphy training on hyperarousal symptoms for childhood survivors of the 2008 China earthquakes. Neuropsychiatr Dis Treat. 2014;10:977-85.

30. Chan SC, Lam TL, Fong KN, Pang MY, Chan CC. Generalization of contextspecific training in individuals with mild cognitive impairment: an eventrelated potential study. Dement Geriatr Cogn Disord Extra. 2016;6:568-79.

\section{Submit your next manuscript to BioMed Central and we will help you at every step:}

- We accept pre-submission inquiries

- Our selector tool helps you to find the most relevant journal

- We provide round the clock customer support

- Convenient online submission

- Thorough peer review

- Inclusion in PubMed and all major indexing services

- Maximum visibility for your research

Submit your manuscript at www.biomedcentral.com/submit
Biomed Central 